\title{
SixTrack Running Environment in Godzilla
}

\author{
R. Tomás
}

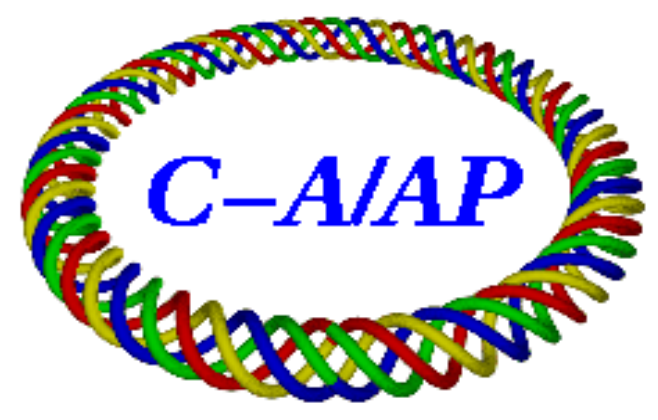

Collider-Accelerator Department Brookhaven National Laboratory Upton, NY 11973 
$\mathrm{CAD} / \mathrm{AP} / 166$

September 4, 2004

\title{
SixTrack Running Environment in Godzilla
}

\author{
R. Tomás
}

\begin{abstract}
A SixTrack [1] running environment was developed in CERN [2] to perform massive tracking campaigns. This environment is highly automated, from the production of the SixTrack input files to the post-processing of the output. It was designed for the CERN's LSF computer cluster, known as LXBATCH. This running environment has been ported to the CAD's parallel multiprocessor computer Godzilla. As many features as possible have been kept. A rudimentary queue system has been designed to replace LSF.
\end{abstract}

\section{Set-up}

The user is assumed to have a large amount (roughly 1Gb) of disk space available for himself. This note will refer to this disk space as the user's SIXTRACK directory, which should be located in his home directory. This is the equivalent directory to the w1 of [2]. Most of the scripts are written for the Korn Shel (ksh). The user needs to check the availability of this shell by, for instance, typing ksh on the prompt. If this shell is not available the user should contact the system administrator.

The Linux version of the SixTrack running environment is located at:

/misc/rap/cvsrelease/tools/SixTrack/SIXTRACK.tar

This file has to be copied to the user's home directory and the command tar - $\mathrm{x}$ SIXTRACK.tar has to be run to extract the files. This will create the necessary entire directory subtree starting with the SIXTRACK directory and the sub-directory COREHOME. The user only needs to do one small editing, in the text file

SIXTRACK/COREHOME/track/sixjobs/GOD

he has to introduce his user-name in the line starting with username=.

\section{Overview}

This environment runs exactly as [2] with three main differences:

- No direct interface with MADX has been provided. The input files fort.3.mother1, fort.3.mother2, fort. 2 and fort. 16 have to be produced by the user and copied to the directory

SIXTRACK/COREHOME/track/v1/s0/<LHCDescrip>,

where LHCDescrip is any string that characterizes the lattice. The files fort. 2 and fort.16 have to be renamed as fort.2_1 and fort.16_1 and gzipped. An example is provided in the directory SIXTRACK/COREHOME/track/v1/s0/2SlicesBB, which is a model of the Blue ring with magnetic errors and four beam-beam interactions.

- The jobs are firstly placed in the file SIXTRACK/COREHOME/track/sixjobs/JOBSINQUEUE and then distributed by running GOD. This will be further discussed.

- There is no available tape-based back-up system and therefore this feature has been removed.

The run environment is a collection of four scripts which are all run from the directory

SIXTRACK/COREHOME/track/sixjobs/

They are normally run in the following order:

1. run_six This puts SixTrack jobs in the JOBSINQUEUE file.

2. GOD This distributes the jobs among the Godzilla processors. Only one job per processor is run at a time for optimizing machine resources.

3. run_join10 This gathers already tracked jobs together and produces combined output files. 
4. run_post This analyses either individually tracked jobs or jobs that have been combined using run_join10.

The scripts should normally be edited beforehand to specify the user's requirements. The user's favorite editing package may be used for this. The following changes are required to all scripts before running:

basedir This is the path to user's SIXTRACK directory.

LHCVers It is set to 1 . The user can change this if he wants to follow a version scheme for archive purposes.

LHCSubVers It is set to 0. Idem as previous item.

LHCDescrip The name of the directory with the input files located at SIXTRACK/COREHOME/track/v1/s0/. In the example given this is 2 SlicesBB.

ista \& iend These are set to 1 in the example. These would allow the use of a large set of input files placed in the same directory with names: fort.2_1, fort.2_2, fort.2_3, and so on.

\section{$3 \quad$ Tidying up after SixTrack}

The tracking takes place in sub-directories below SIXTRACK/tmp. This directory has to be removed after all the jobs have finished for freeing disk space. The file

SIXTRACK/COREHOME/track/sixjobs/JOBSINQUEUE

has to be also removed after all the jobs have finished. Not removing this file would cause the resubmission of the same jobs in the following run.

\section{Concluding remarks}

The precise description of the contents of the different scripts is available in [2]. The differential algebra type of run has not been tested in this new version of the running environment. The user is encouraged to contact the author for questions, bug reports or possible improvements.

\section{Acknowledgements}

Thanks to Yun Luo who dedicated time in successfully testing this environment. Thanks to Frank Schmidt for providing a bug-free version of SixTrack. The author is thankful to Mark Hayes for his comprehensible programming style. Thanks also go to Wolfram Fischer for motivating this work.

\section{References}

[1] F. Schmidt, "SixTrack: Version 3, Single Particle Tracking Code Treating Transverse Motion with Synchrotron Oscillations in a Symplectic Manner, User's Reference Manual" , CERN/SL/94 56 (AP)

(see also http://wwwslap.cern.ch/frs/Documentation/doc.htmlx).

[2] M. Hayes, F. Schmidt, "Run Environment for SixTrack" CERN LHC Project Note 300, July 2002. 